\title{
Shear stress fluctuations in the granular liquid and solid phases
}

\author{
F. Dalton, F. Farrelly, A. Petri, L. Pietronero, L. Pitolli and G. Pontuale \\ Consiglio Nazionale delle Ricerche, Istituto dei Sistemi Complessi, \\ sede di Tor Vergata, Via del Fosso del Cavaliere 100, 00133 Roma, Italy.
}

(Dated: June 10, 2021)

\begin{abstract}
We report on experimentally observed shear stress fluctuations in both granular solid and fluid states, showing that they are non-Gaussian at low shear rates, reflecting the predominance of correlated structures (force chains) in the solid-like phase, which also exhibit finite rigidity to shear. Peaks in the rigidity and the stress distribution's skewness indicate that a change to the force-bearing mechanism occurs at the transition to fluid behavior, which, it is shown, can be predicted from the behavior of the stress at lower shear rates.

In the fluid state stress is Gaussian distributed, suggesting that the central limit theorem holds. The Fiber-Bundle Model with random load sharing effectively reproduces the stress distribution at the yield point and also exhibits the exponential stress distribution anticipated from extant work on stress propagation in granular materials.

PACS numbers: $45.70 .-\mathrm{n}, 05.40 .-\mathrm{a}$
\end{abstract}


Ubiquitous, Granular Media (GM) are important in many fields of human activity and in the natural environment. Their behavior can often be described effectively by phenomenological laws, but physical insight and intuition are often lacking [1, 2]. In particular, a generic friction law for GMs would be a highly desirable achievement [3, 4] both industrially and in the research environment, with implications in other fields, for example earthquake dynamics [5]. Acting against the realization of such a law, however, are the fluctuations and inhomogeneity innate to GMs [6], which primarily arise from the fact that GMs are non-ergodic, dissipative, out-of-equilibrium systems: in dense GMs the kinetic energy can be many orders of magnitude less than the work necessary to overcome gravitational and friction forces, and so the dynamics is limited to the vicinity of metastable, blocked states. Moreover, stress propagation is highly inhomogeneous and anisotropic [6, 7]. When mechanically excited, therefore, the mean behavior can be swamped by the fluctuations (in time, space and ensemble) inherent to the system. These fluctuations carry important information on the microscopic dynamics, and it seems therefore incumbent to investigate the variance of physical quantities, by measuring not only their averages but their full distributions. In fact, such information may be of help in setting up theoretical frameworks or in discriminating between theories with similar predictions for macroscopic average quantities, for example granular friction.

In this Letter we report on the statistical properties of resistance to shear in a dry granular medium. It has long been known [8] that, under increasing shear rate, a granular medium displays a transition from a solid-like (stick-slip) to a fluid-like (sliding) phase. However a complete understanding of this transition seems yet unavailable 9]. Moreover, the statistical properties of macroscopic quantities have never, to our knowledge, been previously investigated in this context (previous time-resolved measurements of stress were performed with different aims in $[10,11,12,13,14]$ among others). Our findings show that the two phases have different statistical signatures unveiling a different origin for the internal stress.

Our experimental set-up consists of a circular Couette cell containing mono-disperse $2 \mathrm{~mm}$ glass beads. An annular plate is driven over the top surface of the channel by a motor via a torsion spring. As the motor winds the spring, the torque on the annular plate eventually exceeds the medium "static friction" $f_{s}$ and so the plate slips. It is similar to the system described in Ref. [14], the main difference being that the medium in the apparatus presented here is free to select its own volume. The inner and outer diameters of the channel are 20 
and $36 \mathrm{~cm}$ respectively and the channel is typically $5 \mathrm{~cm}$ deep. To ensure a granular shearing plane, the annular top plate has a layer of beads glued to its lower surface, though, to avoid individual grains jamming the system at the boundary, this layer of beads does not extend to the full width of the channel. The system is initialized before each experiment by pouring the medium into the channel, then the system is run at a slow velocity $(\simeq 0.01 \mathrm{rad} / \mathrm{s})$ for a long time (of order 1000 revolutions), such that it may approach a stationary state (we have observed that the mean torque approaches a steady state after the order of $10-100$ revolutions). During all the tests reported here, the relative humidity was lower than $40 \%$.

Our measurements from the device consist of the angular position of the annular top plate and the deflection of the torsion spring, sampled at $1 \mathrm{kHz}$ with an error $\simeq 10^{-4} \mathrm{rad}$. Denoting $\theta$ the angular coordinate of the plate in the reference frame of the laboratory, the reaction torque $f_{r}(\leq 0)$ exerted by the system during motion is

$$
f_{r}=I \ddot{\theta}+\kappa\left(\theta-\omega_{0} t\right)
$$

where $I$ is the momentum of inertia of the plate, $\kappa$ is the spring constant and $\omega_{0}$ the driving angular velocity. We identify in particular the static torque, $f_{s}$, which is the maximum torque achieved just before a slip, and a "dynamic" torque $f_{d}$ corresponding to trajectory points for which $\ddot{\theta}=0$. The system displays proportionality between the average static torque and the plate weight, as expected if the Mohr-Coulomb failure criterion is to remain valid [15].

We have investigated the statistics of the instantaneous $f_{\alpha}(\alpha=r, s, d)$ for several different driving velocities and employing springs of different constants. It emerges that at low driving velocities, i.e. in the solid-like, or stick-slip regime, the distribution of $f_{\alpha}$ is not symmetric and displays long tails at large values for all torsion springs [16]. These distributions are shown in figure 1 and, among all the curves tested, are best fitted by a log-normal distribution (also shown, solid lines):

$$
p(f)=\frac{1}{\sqrt{2 \pi} \sigma \cdot\left(f-f_{0}\right)} \exp \left[-\frac{\left(\ln \left(f-f_{0}\right)-\ln \left(\bar{f}-f_{0}\right)\right)^{2}}{2 \sigma^{2}}\right],
$$

with $\left(f>f_{0} \forall f\right)$. It is worth mentioning that the same shape has been found in experiments on polymer films [17] and solid-on-solid friction [18]. This suggests that, despite the different nature of the three systems, similar mechanisms may lie beneath the observed stick-slip behavior. 
When the driving velocity is increased above a critical value $\omega_{c}$, the fluctuations change: The inset to Fig 1 shows the dynamic friction data $f_{d}$ in a comparison between a slowlydriven $(\square)$ and a fast-driven $(+)$ experiment and their curve-fits (solid lines). The fast-driven experiment is clearly less asymmetric, and indeed the best-fit curve obtained indicates a Gaussian fit. From this feature we hypothesize that, during the fluid phase, resistance to shear is dominated by short lived, independent contacts and that the central limit theorem applies (in the presence of dissipation this may not happen even in a pure collisional regime [19]). Evidence for the validity of this hypothesis will be given below. On the contrary, at low velocity, the stress bearing units are constituted by correlated stress chains [6, 7], for which stress does not add independently.

Investigation of the log-normal parameters as functions of the driving velocity show that the average torque is approximately constant at low velocity whereas it increases at high velocity, as argued in previous work [15] and observed in other experiments [20]. Figure 2 shows the mean torque $\left\langle f_{r}\right\rangle$, the rigidity $f_{0}$ (the minimum value of the torque) and the distribution skewness $\gamma$ as functions of the driving velocity for different spring constants. The small diversity in $\left\langle f_{r}>\right.$ between the different torsion springs $(\sim 12 \%)$, with respect to the change in the spring constant (7.5-fold increase), and the absence of a clear trend indicate that the variation is due primarily to configurational differences induced between different series of experiments. This behavior is expected on a theoretical basis and has been observed experimentally [8]. All experiments show that at low velocity the width of the torque distribution is also constant and decreases at high velocity while at the same value of $\omega_{0}, \omega_{c} \simeq 10^{-1} \mathrm{rad} / \mathrm{sec}$, the rigidity and the distribution skewness peak before vanishing. Thus the distribution shape crosses to a symmetric Gaussian, which is the limit form of the log-normal when $\sigma \ll 1$ :

$$
\frac{1}{\sigma^{2}}\left[\ln \left(f-f_{0}\right)-\ln \left(\bar{f}-f_{0}\right)\right]^{2} \simeq\left[\frac{\bar{f}-f}{\left(\bar{f}-f_{0}\right) \sigma}\right]^{2} .
$$

This is the fluidization transition, above which the plate continuously slides.

We have also measured the dependence of the instantaneous torque on the instantaneous plate velocity $\dot{\theta}$. This is shown at the dynamic friction points in the inset to Fig. 2 for a representative experiment. The data show a roughly constant value of the torque for 2 decades of the plate velocity, as in solid friction. As the latter approaches the fluidization value $\omega_{c}$, there is a slow drop followed by a somewhat faster increase, as predicted theo- 
retically [21]. Such behavior has also been observed in some experiments [22] on GMs and model systems [23] and, in Fig. 2 has been fitted by a curve of the form:

$$
f_{d}(\dot{\theta})=f_{0}+\gamma\left(\dot{\theta}-2 \omega_{f} \ln \left(1+\dot{\theta} / \omega_{f}\right)\right)
$$

with $f_{0}=0.63 \mathrm{Nm}, \gamma=0.19 \mathrm{Nms} / \mathrm{rad}, \omega_{f}=0.043 \mathrm{rad} / \mathrm{s}$. Finally, when the velocity becomes comparable or larger than the critical driving velocity $\omega_{c} \simeq 10^{-1} \mathrm{rad} / \mathrm{sec}$, the torque increases almost quadratically $\left(f_{d} \approx \dot{\theta}^{1.9}\right.$ in the figure). From this fact two conclusions can be drawn:

$i)$ : the medium is almost fully fluidized above $\omega_{c}$. Only in this case is resistance to stress expected to increase quadratically with the shear rate [15], In this regime dynamics is dominated by collisions, and from the Gaussian distribution observed we argue that conditions for applying the central limit theorem hold. Moreover, the plate velocity in our experiments can be identified with the shear strain rate up to a proportionality factor.

ii): fluidization occurs intermittently whenever the plate's instantaneous velocity is larger than the critical value, even at low driving. Thus the value of the fluidization velocity can be predicted while driving the system at a much lower velocity. Note, however, that not all slip events reach the high velocity necessary for the fluid state, and so we deduce that for these events, the solid-like phase remains dominant. As for the mean stress as a function of the driving velocity, here there is no evidence of a trend with respect to the spring constant.

The stress distribution observed in the structured (solid-like) phase, Eq. (2), can be reproduced in a robust way by modeling the evolution of stress within the medium as occurring along a finite number of interacting force chains in a stress field. Before describing the model in detail however, it is necessary to understand precisely the behavior which the model must exhibit. In GMs, both compressional and shearing stress propagates in the medium through highly directed chains of grains 6, 7] that are topologically one-dimensional, although branching and crossing become highly probable when the packing fraction increases [6, 24]. These chains form a random network which appears rather rarefied, screening most of the medium volume from stress. The stress sharing among the supporting structures has been measured in granular media in two [6, 25] and three dimensions [24], and has been found to display a long exponential tail for stresses $s$ above the 
mean value, $\bar{s}$. That is, the number of stress chains supporting a stress $s$ is proportional to

$$
\left.n(s)\right|_{s>\bar{s}} \sim \exp [-s / \bar{s}]
$$

This behavior seems quite general, since it has been also observed in a compressed emulsion [26] and in numerical simulations of glasses [27]. Thus the model should also satisfy the constraint of reproducing in a robust way an exponential tail, Eq. (4) in the distribution of the chain loads before the final yielding, besides the log-normal shape of the yield-stress distribution.

To adequately model the stress evolution of the force chains within the medium, perhaps the simplest non-trivial scheme is to consider a bundle of one-dimensional uniform fibers supporting a longitudinal stress. The first such Fiber-Bundle Model (FBM) was introduced 28] and extensively investigated [29, 30] for describing the fracture of textiles. It consists of a bundle of $N$ fibers loaded in parallel, whose individual strength to failure is randomly extracted from some preselected distribution $p(\sigma)$. The present analogy associates a stress chain in the medium with a fiber, and has also been employed for describing the constitutive behavior of compressed GMs [31]. Besides the load sharing law among fibers, we are interested in the distribution of the global failure stress $S$ when the number of fibers $N$ is not large, as results from imaging of force chains would suggest [6, 7, 25]. This corresponds to the distribution of the yield stress of the medium under the increasing elastic shear.

In the primitive FBM the re-distribution of the loads is global, also known as "democratic sharing". That is, as soon as the increasing applied stress exceeds the failure load of one (the weakest) fiber, this breaks and its load is evenly redistributed among all the remaining fibers. If it happens to some of them to be in turn overloaded, they too break and redistribute the load to all the others, and the cycle is repeated until no more fibers break. Then the stress on the system is further increased until the following fiber breaks, and so on. The FBM adopted here assumes instead that whenever a fiber fails its load is re-distributed in random proportions $p \in[0,1]$ and $q=1-p$ among two other fibers chosen randomly from the remaining fibers (Random FBM). In fact the formation of stress supporting chains and the exponential tail in the stress sharing (Eq. (4)) are explained for compressional stress by a simple mechanism (commonly known as the $q$-model [32]) in which the stress exerted on a grain is randomly transmitted to some neighboring grains [32, 33, 34, 35].

Generically speaking, FBMs with $N \rightarrow \infty$ possess rupture at a pre-determined value 
$S_{p}$ set by the initial distribution of fiber thresholds, whereas with $N$ not large, $S_{p}$ assumes an asymmetric distribution for a large class of initial fiber strength distributions $p(\sigma)[29$, 30]. We have performed several simulations exploring the parameter space of the RFBM; principally how different forms for the probability distribution of the fiber strengths affects the behavior of the model. In Fig. 3 the distribution of bundle strengths for a range of initial fiber strength distributions is shown. The result is observed to closely follow the log-normal distribution for a wide variety of input distributions (results for exponential, Gumbel, Weibull and log-normal inputs are shown, but similar results are obtained by other distributions $p(\sigma)$ ). The corresponding fiber load distributions just before the final rupture are shown in the inset to Fig. [3. The final break-down corresponds to the system yield and triggers a slip event.

In all cases the load sharing is characterized by a long exponential tail which, simulations show, is quickly set up after starting to load the system [36]. For exponential, Gumbel and Weibull fiber strengths (amongst others), the fiber-load distribution in RFBM seems to have a robust exponential tail, while for log-normal a stretched exponential is obtained, though with appropriate parameters the stretching exponent $\beta \simeq 1$. If we assume that the overall strength of a force chain would be determined by its weakest element, then the limit distributions for extreme values [37] would be natural choices for the initial fiber strengths distributions (a detailed study of the model with quantitative reconstruction techniques of the stress distribution [38] will be published elsewhere [36]).

Thus, when loaded towards its stability limits, RFBM yields both the internal load sharing, Eq. (4), and the yield stress distribution, Eq. (2), observed in the experiments. It can also in principle consider the formation and destruction of force fibres during shear motion, by including the possibility that fibers "heal" [39], in such a way that in the stick slip regime the system is constantly at the edge of failure, constituting a possible starting point for understanding the similarities observed in the stress distributions in solid-on-solid and polymer film shear experiments. Our results indicate that in the solid regime the system self-organizes in structures, or chains, supporting the stress with an asymmetric distribution about the mean value which can be robustly reproduced by the Fiber-Bundle model with random load sharing. By increasing the driving velocity instantaneous fluidization becomes more and more frequent until the fluid regime is reached, in which the dynamics is collisional and stress distribution is symmetric. We hope that the knowledge of stress fluctuation will 
be of help in improving the theoretical description of the granular solid-liquid transition.

We are grateful to A. Baldassarri and S. Zapperi for pointing out the logarithmic drop of instantaneous friction with velocity. This work was supported by the EU Contract No. ERBFMRXCT980183 and by the FIRB Project RBAU01883Z.

[1] H. M. Jaeger, S. R. Nagel, and R. P. Behringer, Rev. Mod. Phys. 68, 1259 (1996).

[2] P. G. de Gennes, Rev. Mod. Phys. 71, 5374 (1999).

[3] F. Lacombe, S. Zapperi, and H. J. Herrmann, Eur. Phys. Journ. E 2, 181 (2000).

[4] M. O. Robbins, in Jamming and Rheology: Constrained Dynamics on Microscopic and Macroscopic Scales, edited by A. J. Liu and S. R. Nagel (Taylor and Francis, London, 2000).

[5] C. H. Scholz, Nature 391, 37 (1998).

[6] D. Howell, R. P. Behringer, and C. Veje, Phys. Rev. Lett. 82, 5241 (1999).

[7] A. Drescher and G. de Josselin de Jong, J. Mech. Phys. Solids 20, 337 (1972).

[8] R. A. Bagnold, Proc. Roy. Soc. A 295, 219 (1962).

[9] S. Luding, Phys. Rev. E 63, 42201 (2001).

[10] B. Miller, C. O’Hern, and R. P. Behringer, Phys. Rev. Lett. 77, 3110 (1996).

[11] S. Nasuno, A. Kudrolli, and J. P. Gollub, Phys. Rev. Lett. 79, 949 (1997).

[12] S. Nasuno, A. Kudrolli, A. Bak, and J. P. Gollub, Phys. Rev. E 58, 2161 (1998).

[13] R. Hartley and R. P. Behringer, Nature 412, 928 (2003).

[14] F. Dalton and D. Corcoran, Phys. Rev. E. 63, 61312 (2000).

[15] S. B. Savage and M. Sayed, J. Fluid Mech. 142, 391 (1984).

[16] Very recently [40] a system with exactly the same geometry of the experiment presented here has been simulated for the first time. Even in that case the tangential stress distribution has been found to be asymmetric in the solid phase. However a quantitative comparison with the experimental results is difficult since the system is simulated in the absence of gravity and statistical distributions refer to both time and spatial fluctuations.

[17] B. Briscoe, A. Winkler, and M. J. Adams, J. Phys. D: Appl. Phys. 18, 2143 (1985).

[18] A. Johansen, P. Dimon, C. Ellegaard, J. S. Larsen, and H. H. Rugh, Phys. Rev. E 48, 4779 (1993).

[19] A. Puglisi, V. Loreto, U. M. B. Marconi, A. Petri, and A. Vulpiani, Phys. Rev. Lett. 81, 3848 
(1998).

[20] G. Ovarlez, E. Kolb, and E. Clement, Phys. Rev. E 64, R60302 (2001).

[21] H. M. Jaeger, C. h. Liu, S. R. Nagel, and T. Witten, Europhys. Lett. 11, 619 (1991).

[22] M. Lubert and A. de Ryck, Phys. Rev. E 63, 21502 (2001).

[23] F. Heslot, T. Baumberger, B. Perrin, B. Caroli, and C. Caroli, Phys. Rev. E 49, 4973 (1994).

[24] H. A. Makse, D. L. Johnson, and L. M. Schwartz, Phys. Rev. Lett. 84, 4160 (2000).

[25] R. P. Behringer, D. Howell, L. Kondic, S. Tennakoon, and C. Veje, Physica D 133, 1 (1999).

[26] J. Brujić, S. F. Edwards, D. V. Grinev, I. Hopkinson, D. Brujić, and H. A. Makse, Faraday Discuss. 123, 207 (2003).

[27] C. S. O'Hern, S. A. Langer, A. J. Liu, and S. R. Nagel, Phys. Rev. Lett. 86, 111 (2001).

[28] F. T. Peirce, J. Textile Industry 17, 355 (1926).

[29] H. E. Daniels, Proc. R. Soc. A 183, 405 (1945).

[30] M. W. Suh, B. B. Bhattacharyya, and A. Grandage, J. Appl. Prob. 7, 712 (1970).

[31] R. C. Hidalgo, C. U. Grosse, F. Kun, H. W. Reinhardt, and H. J. Herrmann, Phys. Rev. Lett. 89, 205501 (2002).

[32] C.-H. Liu, S. R. Nagel, D. A. Schecter, S. N. Coppersmith, S. N. Majumdar, O. Narayan, and T. A. Witten, Science 269, 513 (1995).

[33] J. E. S. Socolar, Phys. Rev. E 57, 3204 (1998).

[34] U. M. B. Marconi, A. Petri, and A. Vulpiani, Physica A 280, 279 (2000).

[35] P. Claudin, J.-P. Bouchad, M. E. Cates, and J.-P. Wittmer, Phys. Rev. E 57, 4441 (1998).

[36] F. Dalton, A. Petri, G. Pontuale, and A. Virgilii (to be published).

[37] J. Galambos, The asymptotic theory of extreme order statistics (Robert E. Krieger Publishing Company, 1987).

[38] P. N. Inverardi, A. Petri, G. Pontuale, and A.Tagliani, Appl. Math. and Comp. 144, 61 (2003).

[39] R. L. B. Selinger, Z.-G. Wang, W. M. Gelbart, and A. Ben-Shaul, Phys. Rev. A. 43, 4396 (1991).

[40] O. Baran and L. Kondic (2004), cond-mat/0411459. 


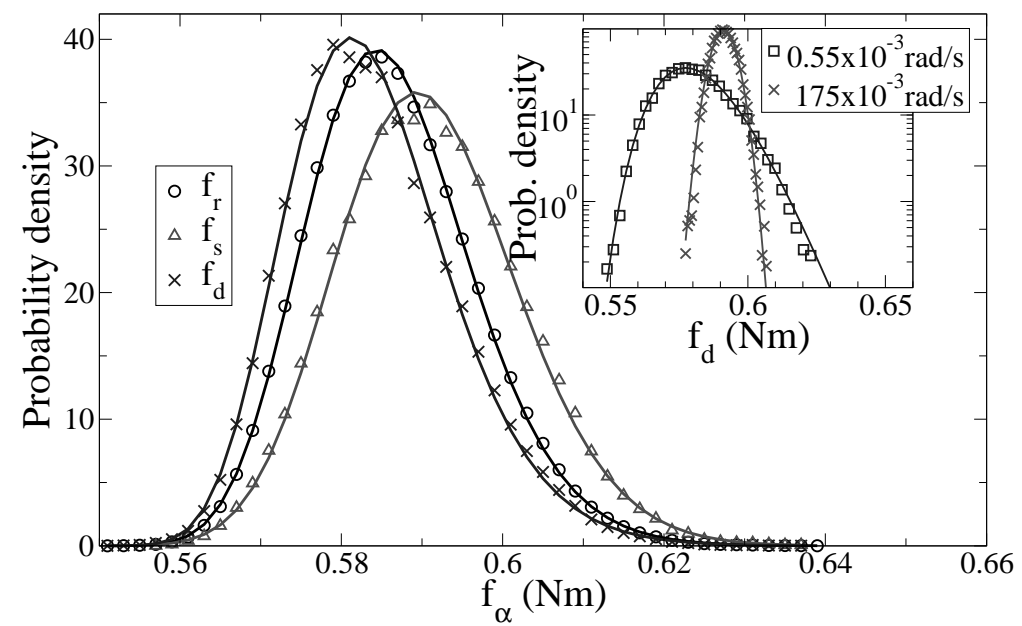

FIG. 1: The distribution of torque in the solid-like regime for $f_{r}(\mathrm{O}), f_{s}(\triangle), f_{d}(\times)$ and their log-normal fits (lines, see Eq. 21). The inset shows two sample experiments, one at slow driving (solid-like regime) $(\square)$, one at fast driving (liquid-like regime) $(\times)$, and their respective log-normal and Gaussian fits, on semi-log axes.

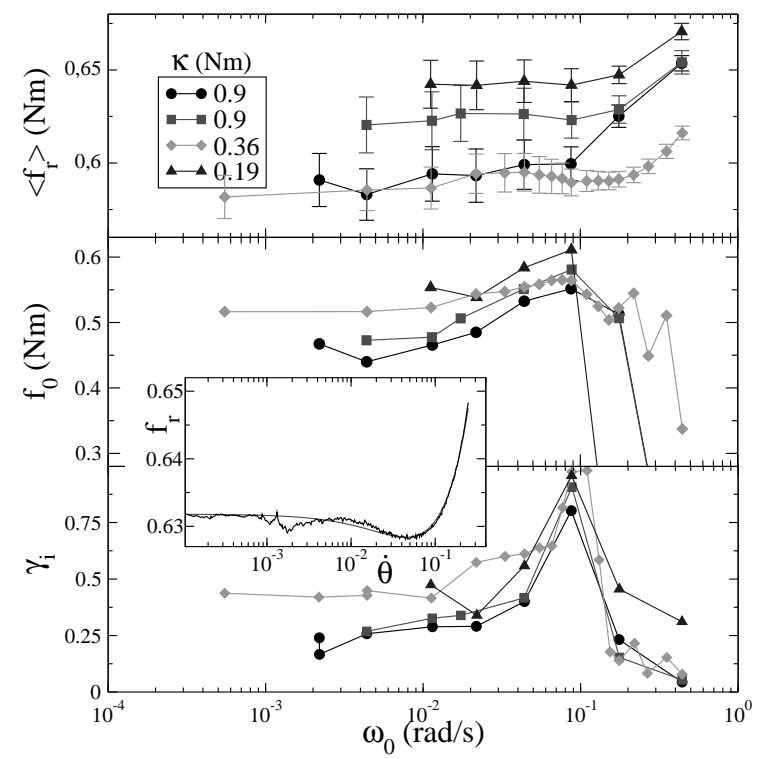

FIG. 2: Fit parameters as functions of the driving velocity for different springs. Top graph: mean torque with standard deviation bars; Center graph: rigidity threshold; Bottom graph: distribution skewness. At the transition, the skewness and the rigidity peak then vanish, indicating that a symmetric Gaussian curve is a good model. Inset: The instantaneous torque as a function of the instantaneous plate velocity at dynamic friction points for a single experiment. 


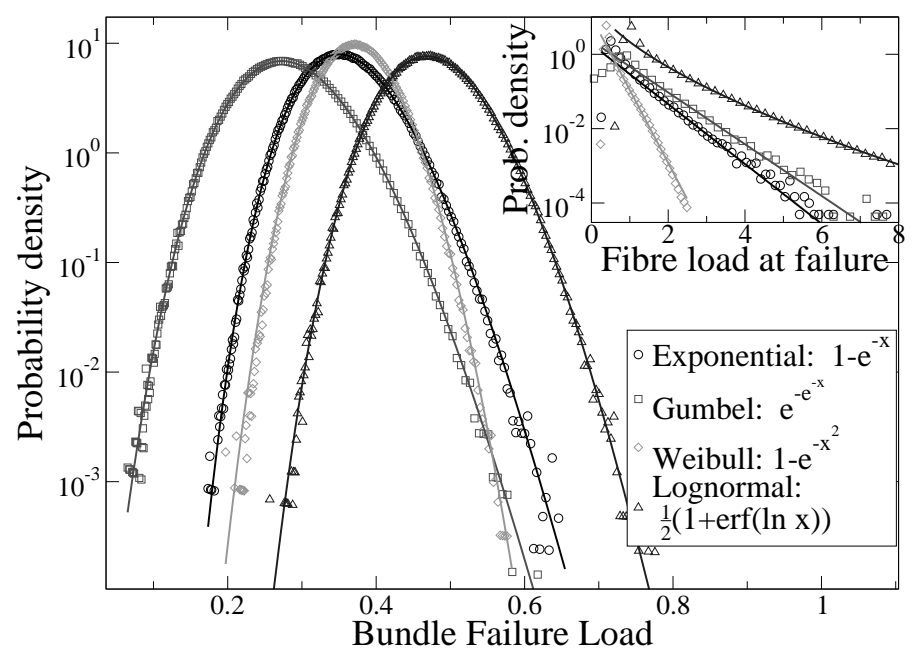

FIG. 3: The bundle strength for a selection of initial fiber distributions with a log-normal fit to each (lines). The inset demonstrates the exponential tail (stretched exponential in the log-normal case) in the distribution of fiber-loads at failure for these same initial distributions. The key to the graph indicates the initial cumulative probability distributions used to create the results (the number of fibers $N=100$ ). 